\title{
CHAOS NEAR A REVERSIBLE HOMOCLINIC BIFOCUS
}

\author{
PABLO G. BARRIENTOS, ARTEM RAIBEKAS, AND ALEXANDRE A. P. RODRIGUES
}

\begin{abstract}
Aвstract. We show that any neighborhood of a non-degenerate reversible bifocal homoclinic orbit contains chaotic suspended invariant sets on $N$-symbols for all $N \geq 2$. This will be achieved by showing switching associated with networks of secondary homoclinic orbits. We also prove the existence of super-homoclinic orbits (trajectories homoclinic to a network of homoclinic orbits), whose presence leads to a particularly rich structure.
\end{abstract}

\section{INTRODUCTION}

A useful indicative of complicated behavior in a differential equation is the presence of homoclinic orbits to a hyperbolic equilibrium point. For instance, in any neighborhood of a Shilnikov homoclinic orbit (a homoclinic orbit connected to a saddle-focus in dimension three) one can find chaos. This chaotic behavior is made clear by means of suspended horseshoes in any number of symbols [19,24]. That is, there are compact invariant hyperbolic sets of the Poincaré return map whose dynamics are topologically conjugate to the Bernoulli shift on $N$-symbols with $N \geq 2$. Similar results were also obtained in the literature $[8,12,20$, 21, 25] for non-degenerate bifocal homoclinic orbits in the non-resonant case.

Given an ordinary differential equation of the type

$$
\dot{x}=f(x), \quad x \in \mathbb{R}^{4}, \quad f \in C^{r}, \quad r \geq 2,
$$

a bifocal homoclinic orbit $\gamma$ is a solution bi-asymptotic to an equilibrium point $O$ such that $D f(O)$ has a pair of eigenvalues $\alpha_{k} \pm i \omega_{k}, k=1$, 2 with $\alpha_{1}<0<\alpha_{2}$ and $\omega_{1} \cdot \omega_{2} \neq 0$. This equilibrium is often called by bifocus. The non-resonant case is given by the saddle index $\delta=\left|\alpha_{1} / \alpha_{2}\right| \neq 1$ and $\gamma$ is said to be non-degenerate if the tangent spaces of the stable and unstable manifold of $O$ have a trivial intersection (containing the flow direction) at any point around the connection $\gamma$. The resonant case $\delta=1$ includes Hamiltonian and reversible systems.

The presence of chaos in any neighborhood of a non-degenerate Hamiltonian bifocal homoclinic orbit was obtained by Devaney in [6]. He proved that, in the critical level set, one can find two-dimensional suspended horseshoes on any number of symbols arbitrarily close to the connection. This result was extended by Lerman [13, 14, 15] for nearby level sets showing the existence of infinitely many suspended two-dimensional horseshoes. In particular, in any neighborhood of a Hamiltonian bifocal homoclinic orbit there are compact invariant sets of the Poincaré return map, which are topologically conjugated to the shift times the identity map (see [2]). 
System (1) is called reversible if there is a linear map $R: \mathbb{R}^{4} \rightarrow \mathbb{R}^{4}$ with $R^{2}=$ id and $\operatorname{dim}($ Fix $R)=2$ such that $f \circ R=-R \circ f$. Recall that Fix $R=\left\{x \in \mathbb{R}^{4}: R x=x\right\}$. A trajectory of (1) is called reversible or symmetric if it is invariant under the involution $R$. The similarity between reversible and Hamiltonian systems has been demonstrated in many cases [5]. For instance, both reversible and Hamiltonian homoclinic orbits are accompanied by a oneparameter family of periodic orbits [7]. However, nothing is known in general about the presence of chaos in a neighborhood of a reversible bifocal homoclinic orbit $\gamma$. Homburg and Lamb studied in [10] this situation under the extra assumption that there is an orbit $\gamma_{a_{0}}$ in the one-parameter family $\gamma_{a}$ of accompanying periodic orbits to $\gamma$ whose stable and unstable manifolds intersect in a reversible bi-asymptotic orbit $\rho_{a_{0}}$ to $\gamma_{a_{0}}$. They concluded that the non-wandering set of the return map describing the dynamics near $\rho_{a_{0}}$ is contained in a set with a lamination of one-dimensional leaves parameterized by a subshift of finite type, that is similar as in the Hamiltonian case.

In this paper,will prove that in any neighborhood of a non-degenerate reversible bifocal homoclinic orbit $\gamma$, we find chaos in an arbitrary number of symbols. We stress that we do not ask for additional hypotheses as the one used in [10]. We will say an invariant set $\Lambda$ of a continuous map $\Pi$ is chaotic on $N$-symbols if $\Pi: \Lambda \rightarrow \Lambda$ is semi-conjugate to the shift on the set $\Sigma_{N}^{+}=\{1, \ldots, N\}^{\mathbb{N}}$ of sequences of $N$-symbols and the periodic orbits of $\Pi$ are dense in $\Lambda$. This notion of a chaotic set is an extension of the so-called chaos in the sense of Block and Coppel $[4,1,16]$. As a consequence of the semi-conjugation we have that $\left.\Pi\right|_{\Lambda}$ has positive entropy, namely $h_{\text {top }}\left(\left.\Pi\right|_{\Lambda}\right) \geq \log N$ and has sensitive dependence on the initial conditions.

Theorem A. For every $N \geq 2$, given any small tubular neighborhood $\mathcal{T}$ of a reversible non-degenerate bifocal homoclinic orbit $\gamma$ of (1), there is a first return map $\Pi$ describing the dynamics near $\gamma$ on $\mathcal{T}$ which has an invariant set $\Lambda_{N}$ chaotic on $N$-symbols.

Devaney showed in [6] that in any tubular neighborhood of a non-degenerate Hamiltonian bifocal homoclinic orbit there are infinitely many secondary homoclinic orbits. That is, other bifocal homoclinic orbits which make several excursions along the primary homoclinic orbit. This result was extended for non-degenerate reversible bifocal homoclinics by Härterich [9] showing that the secondary homoclinics are also reversible and non-degenerate. Thus, in any neighborhood of the primary orbit, we can find a homoclinic network

$$
\Gamma_{N}=\gamma_{1} \cup \cdots \cup \gamma_{N}
$$

associated with the bifocus $O$ composed of $N$ different non-degenerate reversible homoclinic orbits to $O$. The chaotic sets $\Lambda_{N}$ of Theorem A will be obtained by showing the occurrence of switching with respect to the network $\Gamma_{N}$. In other words, we will prove that each sequence associated to the homoclinics of the network is shadowed by a nearby trajectory.

To be precise, consider cross-sections $S_{i}$ transverse to $\gamma_{i}$ and write $\Pi$ for the first return map on the collection of cross-sections $S=S_{1} \cup \cdots \cup S_{N}$. Let $\omega=\left(\omega_{i}\right)_{i \in \mathbb{N}} \in \Sigma_{N}^{+}=\{1, \ldots, N\}^{\mathbb{N}}$. According to [11] (see also [12]), the homoclinic network $\Gamma_{N}$ is called switching if for each tubular neighborhood $\mathcal{U}$ of $\Gamma_{N}$, there exists a flow trajectory $\tau \subset \mathcal{U}$ and a point $x_{\omega} \in \tau$ such that $\Pi^{i}\left(x_{\omega}\right) \in S_{\omega_{i+1}}$ for all $i \geq 0$. We call $x_{\omega}$ the starting point of the realization $\tau$ of $\omega$. 
Following [22], a trajectory $\tau$ is said to be a super-homoclinic orbit to the network $\Gamma_{N}$ if $\tau$ is a bi-asymptotic connection to $\Gamma_{N}$, that is it accumulates on the network $\Gamma_{N}$ in forward and backward time. A super-homoclinic orbit is not a homoclinic loop itself, but its presence implies the existence of large number of the so-called multi-pulse homoclinic loops. We say that the homoclinic network $\Gamma_{N}$ exhibits symmetric super-homoclinic switching if for each $\omega \in \Sigma_{N}^{+}$we find a super-homoclinic orbit realizing $\omega$ with starting point $x_{\omega} \in \operatorname{Fix}(R)$. Since $x_{\omega}$ belongs to $\operatorname{Fix}(R)$, then the super-homoclinic orbit also follows the sequence $\omega$ in backward time. When any prescribed finite path is realized by a reversible homoclinic (resp. periodic) orbit starting in $\operatorname{Fix}(R)$, we say that $\Gamma_{N}$ exhibits symmetric homoclinic (resp. periodic) switching.

In the next main result, we give a description of the orbits in a neighborhood of the non-degenerate reversible homoclinic orbit $\gamma$ :

Theorem B. Under the assumption of Theorem A, every homoclinic network $\Gamma_{N}$ in the tubular neighborhood $\mathcal{T}$ of (1) exhibits symmetric super-homoclinic, homoclinic and periodic switching.

The paper is organized as follows. First, in Section 2 we construct the Poincare return map through the composition of local and semi-global maps. We strongly use the notion of reversibility. After that, in Section 3, the spiralling geometry near the bifocus equilibrium is described. In particular, we state that a disc transverse to the stable manifold of $O$ is mapped, under the first return map, into a spiralling sheet. The geometrical setting is explored in Section 4 to obtain regions on the disk that are mapped by the flow into new spiralling regions containing discs in a position similar to the first one. This allows us to establish the recurrence needed for Section 5, where Theorem A and Theorem B are proven.

Open questions. We finish this section with a couple of open questions. Notice that all the periodic and super-homoclinic orbits found in Theorem A and B are symmetric. Thus the first question, less ambitious, but still interesting is the following.

Question 1: Are there non-symmetric periodic or homoclinic solutions near $\gamma$ ?

Since we are only able to show a semi-conjugacy with the one-sided shift, a natural problem is:

Question 2: Is there a semi-conjugacy with the full two-sided shift on $\mathrm{N}$-symbols?

There are several examples in the literature where switching is proven through the existence of a suspended horseshoe in any neighborhood of the network [11,12]. On the contrary in [11, 18], switching has been shown to result from an attracting network without a suspended horseshoe. From the constructions in Theorem A, the chaotic sets we obtain do not seem to come from suspended horseshoes. But they may arise from the suspension of the so-called partially hyperbolic sets, such as the product of the horseshoe by the identity map. Moreover, in [9] it is proven that homoclinic orbits are actually accumulated by one-parameter families of periodic orbits. This parameter can play the role of the center direction of the partially hyperbolic set. In [10], under extra assumptions, partially hyperbolic sets were shown to exist in reversible systems. Thus our last question is:

Question 3: Can the semi-conjugacy obtained in Theorem A be extended to a conjugacy with a partially hyperbolic set? 


\section{Setting AND Return maps (LOCAl AND global)}

2.1. Setting. Our object of study is the dynamics around a reversible (not necessarily conservative) non-degenerate bifocal homoclinic orbit for which we give a rigorous description here. Specifically, we study a differential equation

$$
\dot{x}=f(x), \quad x \in \mathbb{R}^{4}
$$

where $f: \mathbb{R}^{4} \rightarrow \mathbb{R}^{4}$ is a $C^{2}$-vector field whose flow has the following properties:

(P1) The vector field $f$ is $R$-reversible. That is,

$$
R^{2}=\mathrm{id}, \quad \operatorname{dim}(\operatorname{Fix} R)=2 \text { and } f \circ R=-R \circ f .
$$

In particular, if $x(t)$ is a solution of (2), then so is $R x(t)$.

(P2) The origin $O \in$ Fix $R$ is a bifocus equilibrium. In the reversible case, it follows that the eigenvalues of $D f(O)$ are $\pm \alpha \pm i \omega$ where $\alpha>0$ and $\omega>0$.

(P3) There is a non-degenerate reversible homoclinic orbit $\gamma$ to $O$. That is,

$$
R(\gamma)=\gamma \quad \text { and } \quad \operatorname{dim}\left(T_{\gamma(t)} W^{u}(O) \cap T_{\gamma(t)} W^{s}(O)\right)=1 \quad \text { for all } t \in \mathbb{R} .
$$

In particular, the reversibility implies that $\gamma \cap \operatorname{Fix}(R) \neq \emptyset$.

From now on, $\varphi(t, x)$ denotes the flow of (2) at time $t \in \mathbb{R}$ and initial condition $x \in \mathbb{R}^{4}$. Under assumptions (P1)-(P3), Härterich showed in [9] the following result:

Theorem 2.1 ([9]). In any tubular neighborhood of $\gamma$ there exist infinitely many reversible nondegenerate homoclinic orbits to $O$. Moreover, each homoclinic orbit is accumulated by a one-parameter family of reversible periodic orbits.

We fix a tubular neighborhood $\mathcal{T}$ of $\gamma$ and $N \geq 2$. According to Theorem 2.1 we can find $N$ different reversible non-degenerate homoclinic orbits $\gamma_{i}$ in $\mathcal{T}$. Consider the network

$$
\Gamma_{N}=\gamma_{1} \cup \cdots \cup \gamma_{N} \text {. }
$$

We perform a similar analysis to that of $[9,12]$ and study the first return map over a crosssection transverse to the homoclinic network $\Gamma_{N}$. The flow in a neighborhood of $\gamma$ consists of local and global dynamics. When a trajectory is near the equilibrium its behavior is essentially governed by the linearized vector field. Far from the equilibrium, we use the Tubular Flow Theorem [17] to create a global return map.

2.2. Local dynamics. According to [9], the equation (2) can be linearized near $O$. That is, there exists a neighborhood $V_{O}$ of $O$ such that, if $\left(x_{1}, x_{2}, x_{3}, x_{4}\right)$ is in $V_{O}$, the system (2) is $C^{1}$-orbitally equivalent to the linear system

$$
\left\{\begin{array}{l}
\dot{x}_{1}=-\alpha x_{1}-\omega x_{2}, \\
\dot{x}_{2}=\omega x_{1}-\alpha x_{2}, \\
\dot{x}_{3}=\alpha x_{3}+\omega x_{4}, \\
\dot{x}_{4}=-\omega x_{3}+\alpha x_{4} .
\end{array}\right.
$$


Remark 2.2. The idea behind [9] to linearize the vector field near the origin is Belitskii's theorem [3], which guarantees the $C^{1}-$ linearisation, being enough for our purposes. The refinement of this study (as the stability of elliptic fixed points and more involving bifurcations) requires the use of the normal form described in [23].

Using (3), we may define new bipolar coordinates $\left(r_{s}, \phi_{s}, r_{u}, \phi_{u}\right)$ near the bifocus $O$ :

$$
x_{1}=r_{s} \cos \left(\phi_{s}\right), \quad x_{2}=r_{s} \sin \left(\phi_{s}\right) \quad x_{3}=r_{u} \cos \left(\phi_{u}\right) \quad \text { and } \quad x_{4}=r_{u} \sin \left(\phi_{u}\right) .
$$

The local invariant manifolds are given by

$$
W_{l o c}^{s}(O)=\left\{r_{u}=0\right\} \quad \text { and } \quad W_{l o c}^{u}(O)=\left\{r_{s}=0\right\} .
$$

Near $O$, in bipolar coordinates $\left(r_{s}, \phi_{s}, r_{u}, \phi_{u}\right)$, the dynamics is governed by the differential equations

$$
\dot{r}_{s}=-\alpha r_{s}, \quad \dot{\phi}_{s}=\omega, \quad \dot{r}_{u}=\alpha r_{u} \quad \text { and } \quad \dot{\phi}_{u}=-\omega
$$

whose explicit solutions can be written as

$$
r_{s}(t)=r_{s}(0) e^{-\alpha t} \quad \phi_{s}(t)=\phi_{s}(0)+\omega t \quad r_{u}(t)=r_{u}(0) e^{\alpha t} \quad \phi_{u}(t)=\phi_{u}(0)-\omega t .
$$

2.2.1. Cross sections near to the bifocus equilibrium. Without restriction we can assume that the linear involution $R$ in (2.1) is given by $R\left(x_{1}, x_{2}, x_{3}, x_{4}\right)=\left(x_{3}, x_{4}, x_{1}, x_{2}\right)$, as in [9]. Thus the two-dimensional set of fixed points by $R$ is written in bipolar coordinates as

$$
\operatorname{Fix}(R)=\left\{r_{s}=r_{u}, \phi_{s}=\phi_{u}\right\} .
$$

In order to construct the Poincare map, we consider the three-dimensional return sections near the origin, $\Sigma_{O}^{i n}$ and $\Sigma_{O}^{o u t}$ in $V_{O}$, which are solid tori defined by

$$
\Sigma_{O}^{i n}=\left\{r_{s}=r\right\} \quad \text { and } \quad \Sigma_{O}^{\text {out }}=\left\{r_{u}=r\right\}=R\left(\Sigma_{O}^{\text {in }}\right)
$$

where $r>0$ is chosen sufficiently small such that

$$
\gamma_{i} \cap \Sigma_{O}^{i n}=\left\{q_{i}^{s}\right\} \subset W_{l o c}^{s}(O) \text { for all } i=1, \ldots, N \text {. }
$$

By reversibility we also have that $\gamma_{i} \cap \Sigma_{O}^{\text {out }}=\left\{q_{i}^{u}\right\} \subset W_{l o c}^{u}(O)$ for all $i=1, \ldots, N$. Trajectories starting at $\Sigma_{O}^{i n}$ and $\Sigma_{O}^{\text {out }}$ go outside of $V_{O}$ in negative and positive time, respectively. For convenience we write $\left(\phi_{s}^{\text {in }}, r_{u}^{\text {in }}, \phi_{u}^{\text {in }}\right)$ and $\left(r_{s}^{\text {out }}, \phi_{s}^{\text {out }}, \phi_{u}^{\text {out }}\right)$ for the coordinates in $\Sigma_{O}^{\text {in }}$ and $\Sigma_{O}^{\text {out }}$ respectively. When there is no risk of ambiguity, we write $W^{u / s}$ instead of $W_{l o c}^{u / s}(O)$.

2.2.2. Local flow near to the bifocus. The time of flight inside $V_{O}$ of a trajectory with initial condition $\left(\phi_{s}^{i n}, r_{u}^{i n}, \phi_{u}^{i n}\right) \in \Sigma_{O}^{i n} \backslash W_{l o c}^{s}(O)$ is given by

$$
\frac{1}{\alpha} \ln \left(\frac{1}{r_{u}^{i n}}\right)=-\frac{\ln \left(r_{u}^{i n}\right)}{\alpha} .
$$

Integrating (4), see computations in $[9,12]$, we may define the map $\Pi_{O}: \Sigma_{O}^{\text {in }} \backslash W^{s}(O) \rightarrow \Sigma_{O}^{\text {out }}$ defined by

$$
\left(\phi_{s}^{i n}, r_{u}^{i n}, \phi_{u}^{\text {in }}\right) \mapsto\left(r_{s}^{\text {out }}, \phi_{s}^{\text {out }}, \phi_{u}^{\text {out }}\right)=\left(r_{u}^{\text {in }}, \phi_{s}^{\text {in }}+\frac{\omega}{\alpha} \ln \left(r_{u}^{\text {in }}\right), \phi_{u}^{\text {in }}-\frac{\omega}{\alpha} \ln \left(r_{u}^{\text {in }}\right)\right) .
$$




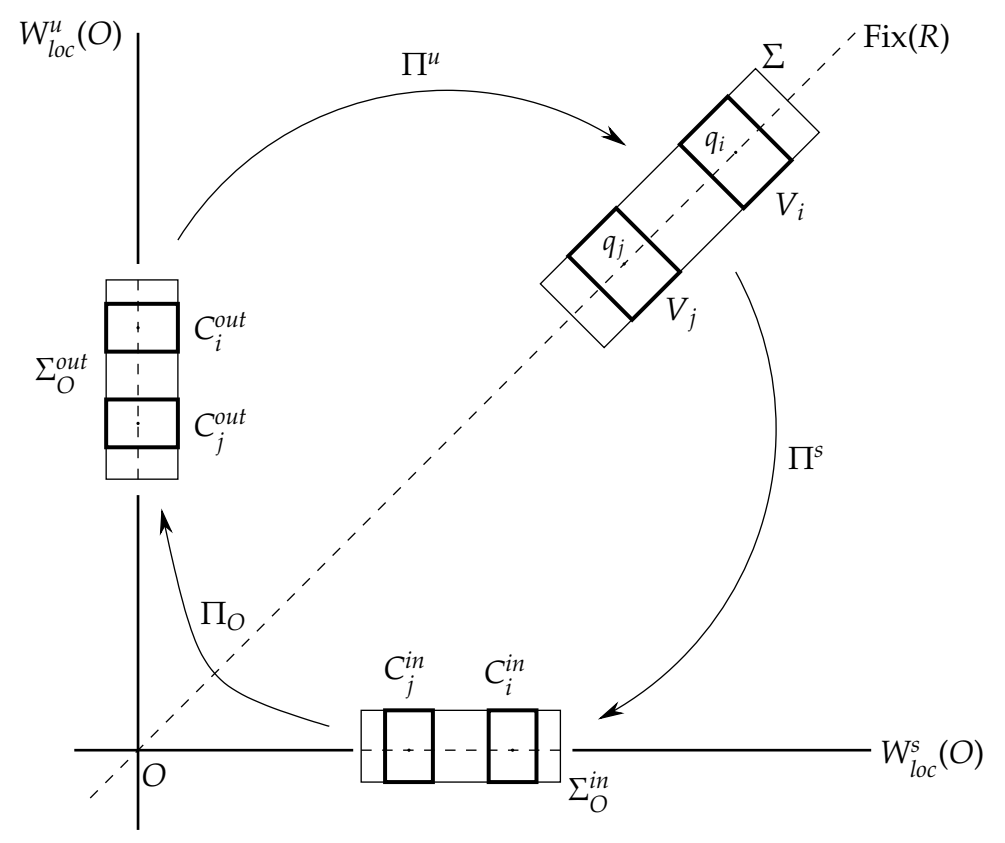

Figure 1. Scheme of the first return map $\Pi=\Pi^{u} \circ \Pi_{O} \circ \Pi^{s}$ on the section $\Sigma$.

2.3. Transitions and global maps. Following Harterich [9], we can make use of the reversibility in the construction of the global Poincaré map by taking a $R$-invariant cross-section $\Sigma$ containing the points

$$
\gamma_{i} \cap \operatorname{Fix}(R)=\left\{q_{i}\right\} \quad \text { for } \mathrm{i}=1, \ldots, \mathrm{N} .
$$

A global map $\Pi^{u}$ between $\Sigma_{O}^{\text {out }}$ and $\Sigma$ is induced by the flow along $\gamma_{i}$. To be more specific, given any neighborhood $V_{i}$ of $q_{i}$ in $\Sigma$, there exists a neighborhood $C_{i}^{\text {out }} \subset \Sigma_{O}^{\text {out }}$ of $q_{i}^{u}$ and a $C^{2}$-map $\tau_{i}: C_{i}^{\text {out }} \rightarrow \mathbb{R}$ such that

$$
\varphi\left(\tau_{i}\left(q_{i}^{u}\right), q_{i}^{u}\right)=q_{i} \quad \text { and } \quad \Pi_{i}^{u}(x):=\varphi\left(\tau_{i}(x), x\right) \in V_{i} \quad \text { for all } x \in C_{i}^{\text {out }} \text { and } i=1, \ldots, N .
$$

By taking the sets $V_{i} \subset \Sigma$ small enough we can obtain that $C_{i}^{\text {out }}$ are pairwise disjoint compact neighborhoods of $q_{i}^{u}$ in $\Sigma_{O}^{o u t}$ for all $i=1, \ldots, N$. Moreover, we can also take $V_{i}$ such that $R\left(V_{i}\right)=V_{i}$. Hence, $\Pi^{u}$ is defined as

$$
\left.\Pi^{u}\right|_{C_{i}^{\text {out }}}=\Pi_{i}^{u} \text { for all } i=1, \ldots, N \text {. }
$$

By reversibility, $\Pi^{s}$ is a semi-global map between $\Sigma$ and $\Sigma_{O}^{\text {in }}$ by means of

$$
\Pi^{s}=R \circ\left(\Pi^{u}\right)^{-1} \circ R \text {. }
$$

Finally, we introduce the Poincaré first return map on $\Sigma$ following the homoclinic network $\Gamma_{N}$ as $\Pi=\Pi^{u} \circ \Pi_{O} \circ \Pi^{s}$ (see Figure 1). Observe that $\Pi$ is actually defined as the composition of three maps: first $\Pi^{s}$ which is well defined from $V=R(V) \subset \Sigma$ to $\Sigma_{O^{\prime}}^{\text {, then }}$

$$
\Pi_{O}: \Sigma_{O}^{\text {in }} \backslash W_{l o c}^{s}(O) \rightarrow \Sigma_{O}^{o u t}
$$


and finally $\Pi^{u}: C \rightarrow \Sigma$ where $V=V_{1} \cup \cdots \cup V_{N} \subset \Sigma$ and $C=C_{1}^{\text {out }} \cup \cdots \cup C_{N}^{\text {out }} \subset \Sigma_{O}^{\text {out }}$. Note that $\Pi$ is of class $C^{r}$ with $r \geq 2$ and it is a reversible map, i.e., $R \circ \Pi \circ R=\Pi^{-1}$.

\section{SPIRALLING GEOMETRY}

In this section we describe the strong spiralling behavior of solutions near $O$. We suggest the reader follows Figure 2.

Let $a \in \mathbb{R}, D$ be a disc centered at $p \in \mathbb{R}^{2}$. A spiral on $D$ around the point $p$ is a smooth curve $S:[0, \infty) \rightarrow D$, satisfying $\lim _{s \rightarrow \infty} S(s)=p$ and such that if $S(s)=(r(s), \phi(s))$ is its expression in polar coordinates around $p$ then

i) $r(s)$ is bounded by two monotonically decreasing maps converging to zero as $s \rightarrow \infty$,

ii) $\phi(s)$ is monotonic for some unbounded subinterval of $[0, \infty)$ and

iii) $\lim _{s \rightarrow+\infty}|\phi(s)|=\infty$.

The notion of spiral may be naturally extended to any set diffeomorphic to a disc.

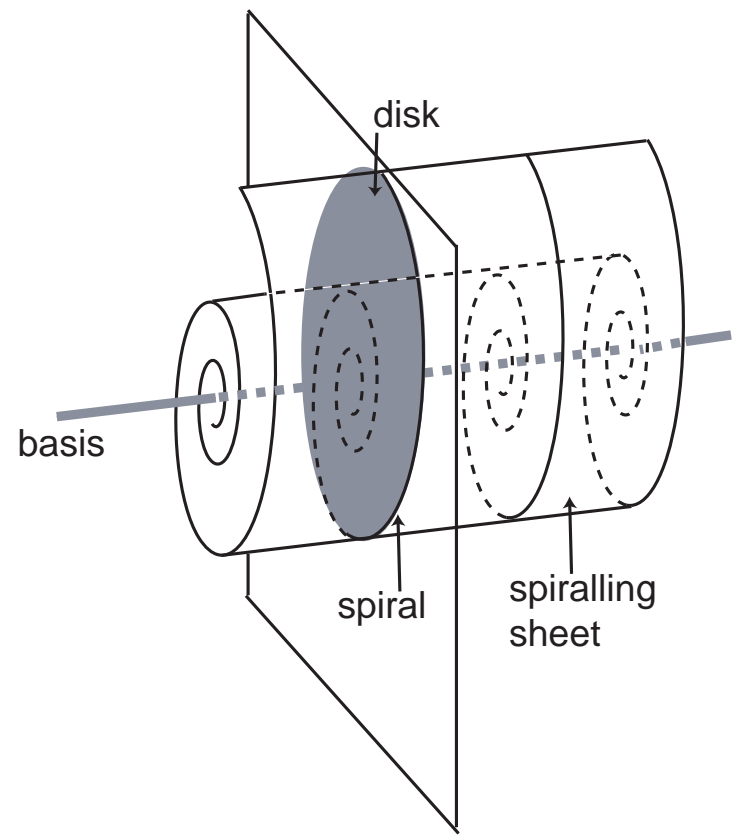

Figure 2. Geometric structures.

A two-dimensional manifold $\mathcal{S}$ embedded in $\mathbb{R}^{3}$ is called a spiralling sheet accumulating on a curve $\mathcal{C}$ if there exist a spiral $S$ around $(0,0)$, a neighborhood $V \subset \mathbb{R}^{3}$ of $\mathcal{C}$, a neighborhood $W_{0} \subset \mathbb{R}^{2}$ of the origin, a non-degenerate closed interval $I$ and a diffeomorphism $\eta: V \rightarrow I \times W_{0}$ such that

$$
\eta(\mathcal{S} \cap V)=I \times\left(S \cap W_{0}\right) \text { and } \mathcal{C}=\eta^{-1}(I \times\{0\}) .
$$

The curve $\mathcal{C}$ may be called the basis of the spiralling sheet. Up to a diffeomorphism, we may think on a spiralling sheet accumulating on a curve as the cartesian product of a spiral and 
a curve. In the present paper, the curve $\mathcal{C}$ lies on the invariant manifolds of $O$. Each cross section to $\mathcal{C}$ intersects the spiralling sheet $\mathcal{S}$ into a spiral. Note also that the diffeomorphic image of a spiralling sheet contains a spiralling sheet.

The following result will be essential in the sequel. It shows that a set diffeomorphic to a disc transverse to $W_{l o c}^{s}(O) \cap \Sigma_{O}^{i n}$ is sent by $\Pi_{O}$ into a spiralling sheet.

Proposition $3.1([9,12])$. For $v>0$ arbitrarily small, let $\Xi: D \subset \mathbb{R}^{2} \rightarrow \mathbb{R}$ be a $C^{1}$ map defined on the disc $D=\left\{(u, v) \in \mathbb{R}^{2}: 0 \leq u^{2}+v^{2} \leq v<1\right\}$ and let

$$
\mathcal{F}^{i n}=\left\{\left(\phi_{s}^{i n}, r_{u}^{i n}, \phi_{u}^{i n}\right) \in \Sigma_{O}^{i n}: \phi_{s}^{i n}=\Xi\left(r_{u}^{i n} \cos \phi_{u}^{i n}, r_{u}^{i n} \sin \phi_{u}^{i n}\right), 0 \leq r_{u}^{i n} \leq v, 0 \leq \phi_{u}^{i n}<2 \pi\right\}
$$

Then the set $\Pi_{O}\left(\mathcal{F}^{i n} \backslash W_{\text {loc }}^{s}(O)\right)$ is a spiralling sheet accumulating on $W_{\text {loc }}^{u}(O) \cap \Sigma_{O}^{\text {out }}$.

\section{THE RECURRENCE}

In Section 2, we have fixed pairwise disjoint compact neighborhood $V_{i}$ of $q_{i}$ in $\Sigma$ for $i=1, \ldots, N$. By means of the global transition maps we have also got pairwise disjoint compact neighborhoods $C_{i}^{\text {out }}=\left(\Pi^{u}\right)^{-1}\left(V_{i}\right)$ of $q_{i}^{u}$ in $\Sigma_{O}^{\text {out }}$ and $C_{i}^{\text {in }}=\Pi^{s} \circ R\left(V_{i}\right)=R\left(C_{i}^{\text {out }}\right)$ of $q_{i}^{s}$ in $\Sigma_{O}^{i n}$ for $i=1, \ldots, N$. We take discs

$$
D_{i} \subset V_{i} \cap \operatorname{Fix}(R) \subset \Sigma \text { centered at } q_{i} \text { for all } i=1, \ldots, N \text {. }
$$

Now, we introduce the local stable un unstable manifolds of $O$ in $\Sigma$ as

$$
W_{i}^{u}=\Pi^{u}\left(W_{l o c}^{u}(O) \cap C_{i}^{\text {out }}\right) \quad \text { and } \quad W_{i}^{s}=\left(\Pi^{s}\right)^{-1}\left(W_{l o c}^{s}(O) \cap C_{i}^{i n}\right) \quad \text { for } i=1, \ldots, N .
$$

Observe that the orbit starting in $W_{i}^{s}$ (resp. $W_{i}^{u}$ ) goes directly to $O$, in forward (resp. backward) time. The proof of the main results needs the following basic result.

Lemma 4.1. If there is an integer $n \geq 0$ such that $x \in W_{i}^{s} \cap \Pi^{n}(\operatorname{Fix}(R))$ or $x \in W_{i}^{u} \cap \Pi^{-n}(\operatorname{Fix}(R))$, then the associated solution is a reversible homoclinic orbit to $O$.

Proof. We prove the case that $x \in W_{i}^{s} \cap \Pi^{n}(\operatorname{Fix}(R))$. The other case is analogous. We have that $\Pi^{-n}(x) \in \operatorname{Fix}(R)$ and hence, by the reversibility, $\Pi^{-2 n}(x) \in W_{i}^{u}$. Thus the orbit associated with $x$ is a homoclinic orbit.

If $\mathcal{R}$ is a measurable set of $\operatorname{Fix}(R) \cap \mathcal{T}$ let us denote by $\mathcal{A}(\mathcal{R})$ its usual area.

Proposition 4.2. For any $k \geq 1, n \in \mathbb{Z}^{k-1}$ and $i \in\{1, \ldots, N\}^{k}$ there exist pairwise disjoints compact sets diffeomorphic to a disc

$$
D_{i j}^{n m} \subset \operatorname{Fix}(R) \quad \text { for all } m \in \mathbb{Z} \text { and } j=1, \ldots, N
$$

such that
i) $D_{i j}^{n m} \subset D_{i}^{n}$,
ii) $\Pi^{k}\left(D_{i j}^{n m}\right) \subset V_{j}$,
iii) there is $q_{i j}^{n m} \in D_{i j}^{n m}$ such that $\Pi^{k}\left(q_{i j}^{n m}\right) \in W_{j}^{s}$ and
iv) there is $\lambda<1$ such that $\mathcal{A}\left(D_{i j}^{n m}\right)<\lambda \cdot \mathcal{A}\left(D_{i}^{n}\right)$. 


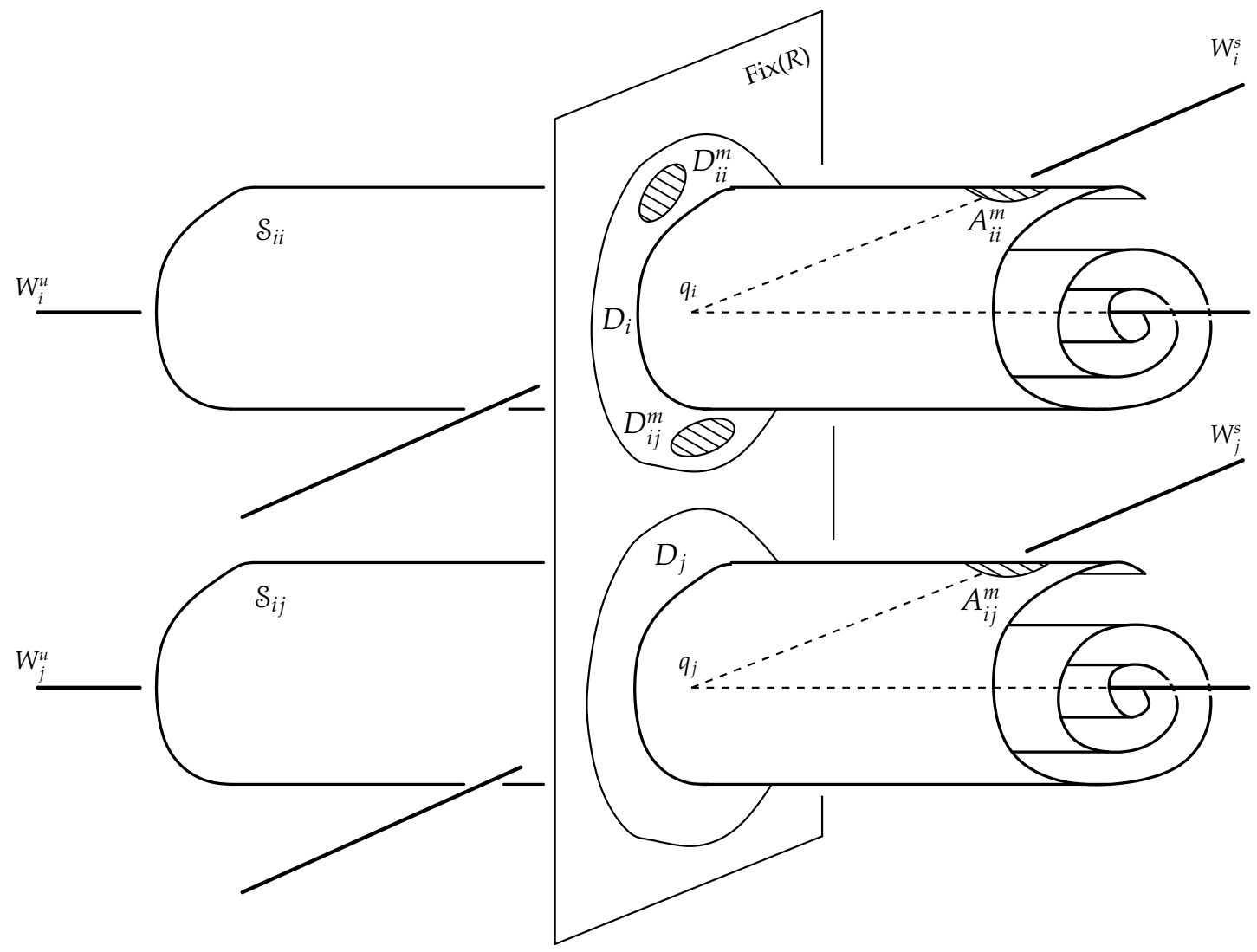

Figure 3. Spiralling geometry for the return map on section $\Sigma$.

Proof. As a consequence of Lemma 4.1 and since $\gamma_{i}$ is a non-degenerate homoclinic orbit, the one-dimensional curves $W_{i}^{s}$ and $W_{i}^{u}$ are both transverse at $\left\{q_{i}\right\}=W_{i}^{s} \cap W_{i}^{u}$ to the two dimensional disc $D_{i} \subset \operatorname{Fix}(R)$. Thus $\Pi^{s}\left(D_{i}\right)$ is a set diffeomorphic to two-dimensional disc transverse to $W_{l o c}^{s}(O) \cap C_{i}^{i n} \subset \Sigma_{O}^{i n}$. Now we will proceed inductively.

According to Proposition 3.1, $\Pi_{O}\left(\Pi^{s}\left(D_{i}\right) \backslash\left\{q_{i}^{s}\right\}\right)=\Pi_{O} \circ \Pi^{s}\left(D_{i} \backslash\left\{q_{i}\right\}\right)$ is a spiralling sheet accumulating on $W_{l o c}^{u}(O) \cap \Sigma_{O}^{\text {out }}$. Hence, $\Pi\left(D_{i} \backslash\left\{q_{i}\right\}\right)=\Pi^{u} \circ \Pi_{O} \circ \Pi^{s}\left(D_{i} \backslash\left\{q_{i}\right\}\right)$ contains a spiralling sheet in $\Sigma$ accumulating on $W_{j}^{u}$ for $j=1, \ldots, N$., which will be denoted by $\mathcal{S}_{i j}$. See Figure 3.

From the quasi-transversality between $W_{j}^{u}$ and $W_{j}^{s}$ (Property (P3)), it follows that $\mathcal{S}_{i j}$ transversally intersect $W_{j}^{s}$ infinitely many times. More precisely, we find a pair of intersection points in each turn of the spiralling sheet. We denote by $Q_{i j}^{m}$ these points in $W_{j}^{s} \cap \mathcal{S}_{i j}$ for $m \in \mathbb{Z}$. From the transversality between $W_{j}^{s}$ and $\mathcal{S}_{i j}$ at $Q_{i j}^{m}$, around these points in the spiralling sheet, we can find pairwise disjoint small compact neighborhoods $A_{i j}^{m} \subset V_{j}$, which are diffeomorphic to a disc, for all $m \in \mathbb{Z}$. Let

$$
D_{i j}^{m}=\Pi^{-1}\left(A_{i j}^{m}\right) \subset D_{i} .
$$


Then, $D_{i j}^{m}$ are pairwise disjoint compact neighborhoods of $q_{i j}^{m}=\Pi^{-1}\left(Q_{i j}^{m}\right) \in D_{i j}^{m}$. Moreover, by decreasing the Area of $A_{i j}^{m}$ we can assume that $\mathcal{A}\left(D_{i j}^{n m}\right)<\lambda \cdot \mathcal{A}\left(D_{i}^{n}\right)$, for some $\lambda<1$. This proves the result for $k=1$. Arguing by induction and repeating the above procedure on each set $\Pi^{s} \circ \Pi^{k-1}\left(D_{i}^{n}\right)$, which up to diffeomorphism is a two-dimensional disc in $\Sigma_{O}^{i n}$ transverse to $W_{l o c}^{s}(O)$, we may conclude the properties stated in the lemma.

Let

$$
K=\bigcap_{k \in \mathbb{N}} \bigcup_{n \in \mathbb{Z}^{k-1}} \bigcup_{i \in\{1, \ldots, N\}^{k}} D_{i}^{n} \text { and } H=\left\{q_{i}^{n}: i \in\{1, \ldots, N\}^{k}, n \in \mathbb{Z}^{k-1}, k \in \mathbb{N}\right\} \text {. }
$$

From Proposition 4.2 it follows that $K$ is an non-empty set and $K \subset \bar{H}$. Moreover, for any pair of sequences $i=\left(i_{k}\right)_{k \in \mathbb{N}} \in \Sigma_{N}^{+}=\{1, \ldots, N\}^{\mathbb{N}}$ and $n=\left(n_{k}\right)_{k \in \mathbb{N}} \in \mathbb{Z}^{\mathbb{N}}$, there is a unique point $x_{i}^{n} \in K$ such that

$$
\left\{x_{i}^{n}\right\}=\bigcap_{k \in \mathbb{N}} D_{i_{1} \ldots i_{k}}^{n_{1} \ldots n_{k-1}} .
$$

Remark 4.3. In fact, any point in $K$ is uniquely identified as above by a pair $(i, n) \in \Sigma_{N}^{+} \times \mathbb{Z}^{\mathbb{N}}$.

Again, Proposition 4.2 and Equation (7) imply that

$$
\Pi^{k}\left(x_{i}^{n}\right) \in V_{i_{k+1}} \quad \text { for all } k \geq 0 .
$$

Since $x_{i}^{n} \in \operatorname{Fix}(R)$, then by the reversibility we also have that $\Pi^{-k}\left(x_{i}^{n}\right) \in V_{i_{k+1}}$ for all $k \geq 1$. This proves the following:

Proposition 4.4. The homoclinic network $\Gamma_{N}=\gamma_{1} \cup \cdots \cup \gamma_{N}$ is switching by reversible trajectories. Moreover, the starting point of the orbit realization can be taken in $K \subset \operatorname{Fix}(R)$ (see (6)).

The next lemma will prove that the flow orbit associated with the point in $K$ approaches the homoclinic network $\Gamma_{N}$ in forward time (by reversibility this also holds in backward time). To shorten the notation, we will say that a sequence $\left(y_{n}\right)_{n}$ converges to a set $A$ if the distance between $y_{n}$ and $A$ goes to zero as $n \rightarrow \infty$.

Lemma 4.5. If $x \in K$ then $\Pi^{k}(x)$ converges to $\left\{q_{1}, \ldots, q_{N}\right\}$ as $|k| \rightarrow \infty$.

Proof. By reversibility, it suffices to prove the lemma for $k \rightarrow \infty$. Let $x \in K$ and since $K$ is identified with $\Sigma_{N}^{+} \times \mathbb{Z}^{\mathbb{N}}$, by means of (7) we get the sequences $i=\left(i_{k}\right)_{k \in \mathbb{N}} \in \Sigma_{N}^{+}$and $n=\left(n_{k}\right)_{k \in \mathbb{N}} \in \mathbb{Z}^{\mathbb{N}}$ such that

$$
\Pi^{k-1}(x) \in A_{k} \stackrel{\text { def }}{=} \Pi^{k-1}\left(D_{i_{1} \ldots i_{k}}^{n_{1} \ldots n_{k-1}}\right) \quad \text { for all } k \geq 1 .
$$

By the inductive construction, $A_{k}$ is a small compact neighborhood of $\Pi^{k}\left(q_{i_{1} \ldots i_{k}}^{n_{1} \ldots n_{k-1}}\right)$, which is diffeomorphic to a two-dimensional disc transverse to $W_{i_{k}}^{s}$ in a spiralling sheet accumulating on $W_{i_{k}}^{u}$. Moreover, we can assume that the radius $r_{k}^{i n}$ of the disc $\Pi^{s}\left(A_{k}\right)$ in $\Sigma_{O}^{i n}$ goes to zero as $k \rightarrow \infty$. According to (5), the radius of the spiraling sheet

$$
\mathcal{S}_{k}=\Pi_{O} \circ \Pi^{s}\left(A_{k} \backslash W^{s}(O)\right)
$$


in $\Sigma_{O}^{\text {out }}$ is $r_{k}^{\text {out }}=r_{k}^{\text {in }}$. Thus, $r_{k}^{\text {out }} \rightarrow 0$ as $k \rightarrow \infty$ and this sequence of spiralling sheets converges to $W_{l o c}^{u}(O)$. This implies that the spiralling sheets in $\Pi^{u}\left(\mathcal{S}_{k}\right)$ converge to $W_{1}^{u} \cup \cdots \cup W_{N}^{u}$. Therefore, $A_{k}$ converges to $\left\{q_{1}, \ldots, q_{N}\right\}$ as $k \rightarrow \infty$ and consequently, $\Pi^{k-1}(x)$ converges to $\left\{q_{1}, \ldots q_{N}\right\}$

\section{Proof of the main Results}

Let $\mathcal{P}$ be the set of points $x \in V \cap \operatorname{Fix}(R)$ such that $\Pi^{n}(x)=x$ for some $n \geq 1$ where $V=V_{1} \cup \cdots \cup V_{N}$. Finally, we will prove our main results:

Proof of Theorem B. From Proposition 4.4 and Lemma 4.5 we conclude that $\Gamma_{N}$ exhibits symmetric super-homoclinic switching. It remains to show symmetric homoclinic and periodic switching.

According to Lemma 4.1 and Lemma 4.2 the flow orbit associated to any point $p \in H$ is a reversible homoclinic orbit. For any $k \geq 1, n=\left(n_{1}, \ldots, n_{k-1}\right) \in \mathbb{Z}^{k-1}$ and $i=\left(i_{1}, \ldots, i_{k}\right) \in$ $\{1, \ldots, N\}^{k}$ we find a point $q=q_{i}^{n} \in H$ such that

$$
\Pi^{j}(q) \in V_{i_{|j|+1}} \quad \text { for }|j| \leq k-1, \quad \Pi^{k-1}(q) \in W_{i_{k}}^{s} \quad \text { and } \quad \Pi^{-(k-1)}(q) \in W_{i_{k}}^{u} .
$$

Thus, any prescribed finite path is realized by a reversible homoclinic orbit starting in Fix $(R)$, and so $\Gamma_{N}$ exhibits symmetric homoclinic switching.

Finally, according to Theorem 2.1, each point $q_{i}^{n} \in H$ is accumulated by a one-parameter family $\left(p_{a}\right)_{a}$ of periodic points $p_{a} \in P$. In particular $H \subset \bar{P}$. Moreover, these periodic orbits follow the homoclinic orbit starting at $q_{i}^{n}$ around a turn before closing. Thus

$$
\Pi^{j}\left(p_{a}\right) \in V_{i_{|j|+1}} \quad|j| \leq k-1, \quad \text { and } \Pi^{k-1}\left(p_{a}\right)=\Pi^{-(k-1)}\left(p_{a}\right),
$$

proving that $\Gamma_{N}$ exhibits symmetric periodic switching.

Proof of Theorem $A$. Consider $\Lambda=K \cup \mathcal{P}$ and let

$$
\Lambda_{N}=\bigcup_{i \in \mathbb{Z}} \Pi^{i}(\Lambda)
$$

Clearly $\Lambda_{N}$ is a $\Pi$-invariant set. Since $H \subset \overline{\mathcal{P}}$ and $K \subset \bar{H}$, then $\Lambda \subset \overline{\mathcal{P}}$, and so the set of periodic points is dense in $\Lambda_{N}$. It remains to prove that $\left.\Pi\right|_{\Lambda_{N}}$ is semi-conjugated to the shift $\sigma$ on the set $\Sigma_{N}^{+}=\{1, \ldots, N\}^{\mathbb{N}}$.

We denote by $\pi: \Lambda_{N} \rightarrow \Sigma_{N}^{+}$the coding map defined by

$$
\pi(x)=\left(i_{n}\right)_{n \in \mathbb{N}} \in \Sigma_{N}^{+} \text {if } \Pi^{k}(x) \in V_{i_{k+1}} \text { for all } k \geq 0 .
$$

Clearly $\pi$ satisfies that $\pi \circ \Pi=\sigma \circ \pi$. From Theorem B, the network $\Gamma_{N}$ is switching and one can take the starting point of the realization in $K \subset \Lambda_{N}$. This implies that $\pi$ is onto (surjective). It is left to see that $\pi$ is also a continuous map.

Fix $x \in \Lambda_{N}, M \in \mathbb{N}$ and for $z \in \Lambda_{N}$ denote by $\pi(z)=\left(j_{n}\right)_{n \in \mathbb{N}}$. It is enough to show that there exists $\delta>0$ such that if $d(z, x)<\delta$, then $j_{n}=i_{n}$ for all $n \leq M$. Now there exists a $\delta_{1}>0$ such that, if $d(z, x)<\delta_{1}$ then $z \in V_{i_{1}}$, and thus $j_{1}=i_{1}$. Since the return map $\Pi$ is continuous 
at $x$, we may find a $\delta_{2}$ such that if $d(z, x)<\min \left\{\delta_{1}, \delta_{2}\right\}$ then $d(\Pi(z), \Pi(x))<\delta_{1}$. As above this implies that $j_{1}=i_{1}$ and $j_{2}=i_{2}$. Proceeding inductively until the $n$-th iterate of $\Pi$ we obtain $\delta=\min \left\{\delta_{1}, \delta_{2}, \ldots, \delta_{n}\right\}>0$ as required. This completes the proof of the semi-conjugation between $\Pi: \Lambda_{N} \rightarrow \Lambda_{N}$ and $\sigma: \Sigma_{N}^{+} \rightarrow \Sigma_{N}^{+}$.

Acknowledgements. P. G. Barrientos was supported by MTM2017-87697-P from Ministerio de Economía y Competividad de España and CNPQ-Brasil. A. Rodrigues aknowledges financial support from Program INVESTIGADOR FCT (IF/00107/2015) and Centro de Matemática da Universidade do Porto. Centro de Matemática da Universidade do Porto (CMUP - UID/MAT/00144/2013) is funded by FCT (Portugal) with national (MEC) and European structural funds through the programs FEDER, under the partnership agreement PT2020. Also, part of this work has been written during the stay of A. Rodrigues in Nizhny Novgorod University, supported by the grant RNF 14-41-00044.

\section{ReFERENCES}

[1] B. Aulbach, and B. Kieninger, On three definitions of chaos, Nonlinear Dyn. Syst. Theory 1, 2337, 2001

[2] P. G. Barrientos, S. Ibañez, and J. A. Rodríguez, Robust cycles unfolding from conservative bifocal homoclinic orbits 31 4, 546-579, 2016

[3] G. R. Belitskii, Functional equations and conjugacy of local diffeomorphisms of finite smoothness class, Funkcional. Anal. i Prilozen, 7, 17-28, 1973

[4] L.S. Block, and W. A. Coppel, Dynamics in One Dimension, Springer Lecture Notes, 1513, Springer Verlag, Berlin, 1992

[5] R. L . Devaney, Reversible Diffeomorphisms and Flows, Transactions of the American Mathematical Society, 218, 89-113, 1976

[6] R. L. Devaney, Homoclinic orbits in Hamiltonian systems, J. Diff. Equations, 21, 431-438, 1976

[7] R. L. Devaney, Blue sky catastrophes in reversible and Hamiltonian systems, Ind. Univ. Math. J., 26, 247-263, 1977

[8] A.C. Fowler, and C. T. Sparrow, Bifocal homoclinic orbits in four dimensions. Nonlinearity 4, 1159-1182, 1991

[9] J. Harterich, Cascades of reversible homoclinic orbits to a bi-focus equilibrium, Physica D, 112, 187-200, 1998

[10] A. J. Homburg, and J. Lamb, Symmetric homoclinic tangles in reversible systems, Ergodic Theory Dynam. Systems 26, 1769-1789, 2006

[11] A. J. Homburg, and J. Knobloch, Switching homoclinic networks, Dynamical Systems: an International Journal, Vol. 25 (3), 351-358, 2010

[12] S. Ibáñez, and A.A.P. Rodrigues, On the dynamics near a homoclinic network to a bifocus: switching and horseshoes, Int. J. Bifurcation Chaos 25, 1530030, 2015

[13] L. Lerman, Complex dynamics and bifurcations in a Hamiltonian system having a transversal homoclinic orbit to a saddle focus, Chaos. 1, 174-180, 1991

[14] L. Lerman, Homo- and heteroclinic orbits, hyperbolic subsets in a one-parameter unfolding of a Hamiltonian system with heteroclinic contour with two saddle-foci, Regul Khaoticheskaya Din. 2, 139-155, 1997.

[15] L. Lerman, Dynamical phenomena near a saddle-focus homoclinic connection in a Hamiltonian system, J Statist Phys., 101, 357-372, 2000

[16] A. Medio, M. Pireddu and F. Zanolin, Chaotic dynamics for maps in one and two dimensions: a geometrical method and applications to economics. International Journal of Bifurcation and Chaos 19, 10 3283-3309, 2009.

[17] J. Palis, and W. Melo, Geometric Theory of Dynamical Systems : An Introduction, Springer, 1982

[18] A.A.P. Rodrigues, Is there switching without suspended horseshoes?, Bull. Soc. Port. of Maths, 2016

[19] L. P. Shilnikov, A case of the existence of a denumerable set of periodic motions, Sov. Math. Dokl. 6, 163-166, 1965 
[20] L. P. Shilnikov, The existence of a denumerable set of periodic motions in four dimensional space in an extended neighbourhood of a saddle-focus, Sovit Math. Dokl., 8(1), 54-58, 1967

[21] L. P. Shilnikov, A contribution to the problem of the structure of an extended neighborhood of a rough equilibrium state of saddle-focus type,, Math. USSR Sb., 10, 91-102, 1970

[22] L. P. Shilnikov and D. Turaev, Super-homoclinic orbits and multi-pulse homoclinic loops in Hamiltonian systems with discrete symmetries. Regul. Khaoticheskaya Din 2, 126-138, 1997

[23] L. P. Shilnikov, A. L. Shilnikov, D. V. Turaev, and L. O. Chua, Methods of qualitative theory in nonlinear dynamics (Part 1), World Scientific Publishing Co., 1998.

[24] C. Tresser, About some theorems by L. P. Shilnikov, Ann. Inst. H. Poincaré, 40, 441-461, 1984

[25] S. Wiggins, Introduction in Applied Nonlinear Dynamical Systems and Chaos, Springer, 1990

Instituto de Matemática e Estatística, UFF

Rua Prof. Marcos Waldemar de Freitas Reis, s/N, Niterói, Brazil

E-mail address: pgbarrientos@id.uff.br

E-mail address: artem@mat.uff.br

Centro de Matemática da Universidade do Porto

Faculdade de Ciências da Universidade do Porto

Rua do Campo Alegre 687, 4169-007 Porto, Portugal

E-mail address: alexandre.rodrigues@fc.up.pt 